\title{
The functions of tumor suppressor PTEN in innate and adaptive immunity
}

\author{
Lang Chen ${ }^{1}$ and Deyin Guo ${ }^{2}$
}

The tumor suppressor phosphatase and tensin homolog (PTEN) is a lipid and protein phosphatase that is able to antagonize the PI3K/AKT pathway and inhibit tumor growth. PTEN also possesses phosphatase-independent functions. Genetic alterations of PTEN may lead to the deregulation of cell proliferation, survival, differentiation, energy metabolism and cellular architecture and mobility. Although the role of PTEN in tumor suppression is extensively documented and well established, the evidence for its roles in immunity did not start to accumulate until recently. In this review, we will focus on the newly discovered functions of PTEN in the regulation of innate and adaptive immunity, including antiviral responses.

Cellular \& Molecular Immunology (2017) 14, 581-589; doi:10.1038/cmi.2017.30; published online 26 June 2017

Keywords: adaptive immunity; innate immunity; PTEN; tumor suppressor

\section{PTEN AND TUMOR SUPPRESSION}

In 1997, two groups independently found that there is a tumor suppressor gene located on chromosome $10 \mathrm{q} 23 .{ }^{1,2} \mathrm{Li}$ et al. ${ }^{1}$ named the gene PTEN, abbreviated from phosphatase and tensin homolog (PTEN) deleted on chromosome 10, which is mutated in human brain, breast and prostate cancer. Steck et al. called it MMAC1 and found that its protein had motifs with significant homology to the catalytic domain of protein phosphatases and to the cytoskeletal proteins, tensin and auxilin. Currently, it is well established that PTEN is a bi-functional phosphatase, and its substrates can be lipids or proteins. $^{3}$

The major biological function of PTEN relies on its phosphatase activity, which dephosphorylates phosphatidylinositol 3,4,5-trisphosphate (PtdIns(3,4,5)P3) to $\operatorname{PtdIns}(4,5) \mathrm{P} 2$, therefore inhibiting the phosphatidylinositide 3-kinase (PI3K) signaling pathway. The PI3K signaling pathway plays important roles in regulating cell metabolism, survival, proliferation, apoptosis, growth and migration. Thus, PTEN exerts tumor suppressive functions by suppressing the PI3K pathway. ${ }^{4}$

Somatic loss-of-function mutations of PTEN can contribute to or can drive a malignant phenotype in a variety of human cancers, including breast cancer, endometrial carcinoma, glioblastoma multiforme, and skin and prostate cancers. There are 1478 unique mutations of the PTEN gene that have been found in tumor cells (http://cancer.sanger.ac.uk/cosmic/search? $\mathrm{q}=\mathrm{pten})$. Some mutations can even directly change the conformation of PTEN or can impair its phosphatase activity. Researchers have also discovered that mutation of one of the PTEN alleles could affect the function of PTEN. The most typical pathology induced by PTEN mutation is PTEN hamartoma tumor syndrome (PHTS). ${ }^{5}$ PHTS confers an increased risk for specific malignancies, mostly breast, thyroid, renal and endometrial cancers.

The role of PTEN as a tumor suppressor gene has been well established, ${ }^{4,6,7}$ and new substrates and functions of PTEN have also been reported. Recently, numerous studies have demonstrated that many proteins involved in immune signaling pathways and immune cell development are regulated by PTEN, indicating that PTEN also plays critical roles in immunological activities. These findings reveal that a functional bridge may exist between PTEN and its ability to drive tumor suppression as well as immune regulation. In this review, we will highlight the functions and regulation of PTEN in adaptive and innate immunity, including antiviral responses.

BIOCHEMICAL PROPERTIES AND FUNCTIONS OF PTEN The PTEN gene maps to chromosome 10q23 in humans and is usually expressed as a 403-aa protein. Recently, a translational variant, PTEN-Long (PTEN-L), has been described that has a

\footnotetext{
${ }^{1}$ Department of Immunology, School of Basic Medical Sciences, Wuhan University, Wuhan 430071, China and ${ }^{2}$ School of Medicine (Shenzhen), Sun Yat-sen University, Guangzhou 510080, China Correspondence: Dr D Guo, PhD, School of Medicine (Shenzhen), Sun Yat-sen University, Zhongshan Erlu 74, Yuexiu District, Guangzhou 510080, China. E-mail: guodeyin@mail.sysu.edu.cn

Received: 5 February 2017; Revised: 10 April 2017; Accepted: 10 April 2017
} 
173-residue N-terminal extension (NTE) produced by translating from an upstream non-canonical in-frame start site CUG. ${ }^{8}$ PTEN has four functional domains, including an N-terminal phosphatase domain (aa 1-185), a C2 domain (aa 186-349), a C-tail (carboxyl-terminal) domain and a PDZ-BD (PDZ-binding) domain ${ }^{9}$ (Figure 1a).

The N-terminal domain of PTEN, also the phosphatase domain, is homologous to tensin and auxilin and contains a phosphatase active site: HCxxGxxR. The structure of this domain consists of a central five $\beta$-sheets and two $\alpha$-helices on one side and four $\alpha$-helices on the other side (Figure 1b). Compared with the vaccinia H1-related phosphatase and Y-specific protein tyrosine phosphatases (PTPases), the PTEN active-site pocket is both wider and deeper, making it able to accommodate larger substrates such as $\operatorname{PtdIns}(3,4,5) \mathrm{P} 3$ and some proteins. The N-terminal domains can also interact with each other and mediate dimer formation of PTEN. ${ }^{9}$

The PTEN C2 domain is structurally similar to the C2 domains of phospholipase $\mathrm{C} \delta 1$, phospholipase A2 and protein kinase $\mathrm{C}$ beta (PKC $\beta$ ), which can bind to phospholipid vesicles and regulate the subcellular localization of PTEN. In addition, the C2 domain is important for PTEN dimer formation, although it does not directly associate with the dimer interface of PTEN. The C2 domain also interacts with the phosphatase domain of PTEN and regulates its phosphatase activity. ${ }^{9}$

a

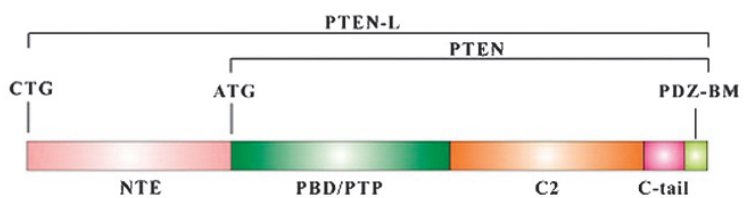

b
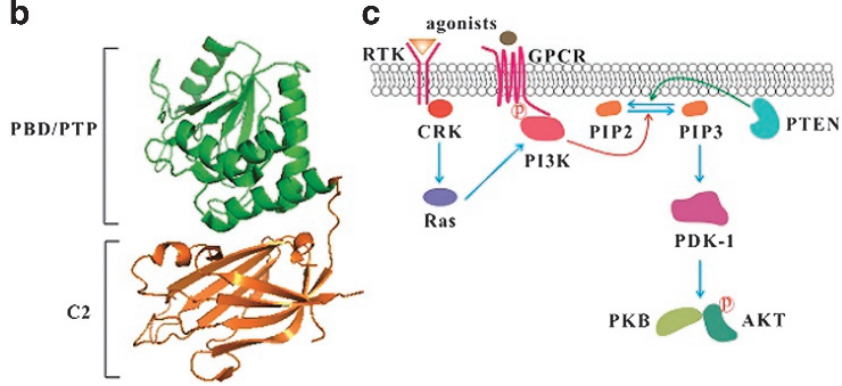

Figure 1 Protein structure of PTEN and its function in antagonizing the PI3K/AKT pathway. (a) Domains of the PTEN protein. NTE, $\mathrm{N}$-terminal extension in PTEN-Long; PBD, Ptdlns(4,5)P2-binding domain; PTEN-L, PTEN-Long, a translational variant of PTEN; PTP, protein tyrosine phosphatase. (b) Crystal structure of PTEN PDB/ PTP and C2 domain. Green indicates N-terminal domains consisting of PDB and PTP; orange represents the C2 domain. (c) Sketch map of PTEN in the de-phosphorylation of $\operatorname{Ptdlns}(3,4,5)$ P3 and the antagonism of the PI3K/AKT signaling pathway. Agonists including hormones, growth factors or cytokines that can bind with their receptors, such as G-protein-coupled receptors (GPCRs), and receptor tyrosine kinases (RTKs), and initiate PI3KAKT signaling pathway, while PTEN can dephosphorylate the key molecule PtdIns(3,4,5)P3 to produce PIP2 (Ptdlns(4,5)P2) and inhibit signal transduction.
The C-tail domain is a disordered domain in the PTEN monomer but is well folded in the PTEN dimer. Moreover, the C-tail domain is important for PTEN activity, stability, homodimer formation and post-translational modification, such as ubiquitination and sumoylation. Phosphorylation of the C-tail domain reduces PTEN activity. ${ }^{10}$

PDZ-BD domains are found in numerous proteins, such as Vangl2, which normally mediate protein-protein interactions through binding to proteins containing PDZ domains. ${ }^{11}$ The PTEN PDZ-BD (aa 384-403) domain is a functional type 1 PDZ-binding motif (PDZ-BM). ${ }^{12}$ The C-terminal eight residues 'HTQITKVT' of PTEN are important for specific recognition of PDZ domains, and the phosphorylation of proximal residues of the PTEN C-terminal tail (Ser380/Thr382/Thr383) can diminish binding to PDZ domains, likely by maintaining the $\mathrm{C}$ terminus of PTEN in a closed conformation. ${ }^{13,14}$

Mostly, PTEN can function as a phosphatase to adjust the phosphorylation status of lipids and proteins, thereby regulating their functions. The dephosphorylation activity is determined by the structure of the $\mathrm{N}$-terminal domain of PTEN but is regulated by its C2 and C-tail domains, which are enhanced upon homodimer formation. In solution, PTEN can switch between open and closed states, and phosphorylation of the C-tail favors a closed conformation. ${ }^{15}$ In addition, PTEN can also interact with proteins through C-2 and PDZ-BD domains, which have a more extensive function. ${ }^{12}$

PTEN is located both in the cytoplasm and nucleus and can be secreted from cells. The optimal substrate of PTEN is PtdIns $(3,4,5) \mathrm{P} 3$ in the cytoplasm. ${ }^{4} \operatorname{Ptd} \operatorname{Ins}(3,4,5) \mathrm{P} 3$ is a phospholipid that resides on the plasma membrane, the level of which increases rapidly following physiologic stimulation. Normally, PTEN can translocate to the cytoplasmic side of the plasma membrane and can dephosphorylate PtdIns $(3,4,5) \mathrm{P} 3$ to PtdIns $(4,5) \mathrm{P} 2$ to inhibit the PI3K signaling pathway in cells, therefore significantly affecting cell behavior (Figure 1c). PtdIns(4,5)P2 is not only a product of PtdIns(3,4,5)P3 via dephosphorylation by PTEN but also a requirement for PTEN membrane binding. ${ }^{15}$ In addition, binding of PTEN with the plasma membrane is also regulated by the phosphorylation status of PTEN. ${ }^{16-18}$ This interaction is delineated in more detail in the following sections.

Increasing evidence indicates that PTEN can function in the nucleus and can play an important role in transcriptional regulation, chromosome stability, DNA repair and cell cycle arrest. There is no evidence proving that PTEN has a nuclear localization signal sequence and its nuclear localization may be regulated by different chemical modifications, such as phosphorylation, ubiquitylation and SUMOylation. 19,20

Within the nucleus, PTEN can act as a phosphatase or can bind with other proteins to participate in different nuclear processes. cAMP response element-binding protein (CREB), a cellular transcription factor, has been shown to be a nuclear substrate of PTEN in vivo and in vitro. ${ }^{21}$ Its phosphorylation could be enhanced independently of PI3K/AKT signaling when PTEN is deleted. PTEN could regulate gene transcription and cell growth in a CREB-mediated manner. ${ }^{21}$ Despite the 
de-phosphorylation functions of PTEN, PTEN can bind proteins through different domains and can function in a phosphatase-independent manner. Shen et al. ${ }^{22}$ found that nuclear PTEN can bind with centromeric protein C, an integral component of the kinetochore, through its C-tail domain and thus participate in guarding chromosomal integrity. In the nucleus, PTEN can recruit Rad51 to the DNA replication fork and can regulate DNA replication progression. In addition, deletion of PTEN can initiate a replication stress cascade and thus regulate the cell cycle. ${ }^{23}$

PTEN-L is a secretory translational variant. It has an additional 173 aa at the $\mathrm{N}$ terminus of PTEN. The NTE of PTEN-L is intrinsically disordered. Moreover, it harbors a secretion signal sequence and a poly-arginine stretch as a signal sequence for entering cells, similar to the poly-basic residues of the human immunodeficiency virus (HIV) trans-activator of transcription (TAT) protein. ${ }^{8}$

Functionally, PTEN-L is fivefold more effective at binding to its substrate PtdIns $(3,4,5) \mathrm{P} 3$ than PTEN. ${ }^{24}$ However, PTEN can be exported in exosomes. ${ }^{25}$ Its recruitment to exosomes needs an adaptor protein, NEDD4 family-interacting protein 1 (Ndfip1), and the ubiquitination of K13 by neural precursor cell expressed developmentally downregulated protein 4-1 (NEDD4-1). The PTEN in exosomes is finally internalized by recipient cells to exert phosphatase activity. Discovery of the secretion and intercellular trafficking of PTEN-L and PTEN holds promise for the development of therapeutics that utilize external PTEN proteins to treat PTEN-deficient tumors.

\section{REGULATION OF PTEN FUNCTIONS}

As discussed above, PTEN plays important roles in both tumor suppression and immune responses. PTEN itself is also tightly regulated by other factors, such as miRNAs and transcriptional and post-translational modifications.

Aberrant methylation of the PTEN promoter can alter PTEN expression. ${ }^{26,27}$ Other known transcriptional regulatory factors include sal-like protein 4 (SALL4), which represses PTEN transcription through a histone deacetylase to the PTEN locus, transacting EMT transcriptional factor SNAIL and the oncogenic factor inhibitor of DNA binding 1 (ID1). ${ }^{3}$

The normal functions of PTEN can be inhibited by redox. ${ }^{28,29}$ There is evidence to suggest that DJ-1, a redoxactive protein, can detoxify NO via transnitrosylation to PTEN, decreasing its phosphatase activity and promoting cell survival. Dysfunctional DJ-1 in Parkinson's disease patients could contribute to neurodegenerative disorders, which are caused by its failure of transnitrosylation to PTEN. ${ }^{28}$ Other peroxides, such as $\mathrm{H}_{2} \mathrm{O}_{2}$ and $\mathrm{pbV}$, can also inhibit PTEN via reversible disulfide formation between $\mathrm{C} 124$ and C71, resulting in small structural changes. ${ }^{29}$

Phosphorylation or dephosphorylation is another regulatory mediator of PTEN function, especially phosphorylation in the $\mathrm{C} 2$ and C-tail domains. Phosphorylation of the PTEN tail inactivates its function by transforming it into a closed conformation. Conversely, dephosphorylation of the PTEN tail favors a more open conformation that allows subsequent dimerization. These phosphorylation sites include Ser370, Ser380, Thr382, Thr383 and Ser385. ${ }^{30,31}$ The phosphorylation of Ser229, Thr232, Thr319 and Thr321 in the PTEN C2 domain regulates its subcellular localization and PTENmediated chemotaxis. ${ }^{32}$ Tyr336 phosphorylation is related to PTEN degradation. ${ }^{33}$ In addition, Tyr240 and Tyr315 phosphorylation could help to maintain phosphatase function of PTEN. ${ }^{34,35}$ Bolduc et al. ${ }^{16}$ found that upon phosphorylation of the 380-385 Ser/Thr cluster, the PTEN C-terminal modified tail clamps down intramolecularly on the $\mathrm{C} 2$ domain, preventing PTEN from binding to the plasma membrane and reducing its catalytic action toward PIP3. Nguyen also proved that the phosphatase catalytic site forms the membrane-binding regulatory interface and interacts with the inhibitory phosphorylated C-terminal tail. ${ }^{17}$

The effect of SUMOylation on PTEN function has also been studied. PTEN can be covalently modified by SUMO1 at both K266 and K254. SUMOylation of K266 is closely related to PTEN membrane localization, whereby it facilitates PTEN binding to the plasma membrane. ${ }^{36}$ SUMOylation of PTEN K254 regulates its nuclear localization. After exposure to genotoxic stress, SUMO-PTEN was rapidly excluded from the nucleus, and this process depended on the phosphorylation of PTEN T398 by ataxia telangiectasia mutated. ${ }^{20,37}$

Acetylation can also regulate PTEN activity. It has been reported that PTEN is acetylated on K125 and K128 by PCAF, on K163 by HDAC6, and on K402 by CBP..$^{38-40}$ Among them, K125 and K128 are located at the catalytic center of PTEN. Their acetylation could downregulate the catalytic activity of PTEN. ${ }^{38}$ K163 acetylation could inhibit the interaction of the PTEN C-tail domain with other domains of PTEN, resulting in PTEN membrane translocation. ${ }^{39} \mathrm{~K} 402$ is located at the PDZ-BD domain of PTEN. CBP and SIRT1 are the major acetyltransferase and deacetylase involved in controlling K402 acetylation. Acetylation of K402 affects PTEN binding with other proteins through the PDB-BD domain. ${ }^{40}$

Ubiquitination of PTEN also affects its activity. There are at least five lysine residues that may be ubiquitinated: Lys13, Lys48, Lys66, Lys80 and Lys289.41 Monoubiquitination can influence the accumulation of PTEN in the nucleus. Polyubiquitination of PTEN leads to its degradation by the proteasome complex. In addition, different ubiquitinase and deubiquitinase enzymes control different aspects of PTEN properties. For instance, OTU deubiquitinase 3 depolyubiquitylates and stabilizes $\mathrm{PTEN},{ }^{42}$ while PI3K/AKT phosphorylates makorin ring finger protein 1 (MKRN1) to promote PTEN degradation via its subsequent uniquitination. $^{43,44}$ Other E3 ubiquitin ligases, such as NEDD4-1, $\mathrm{X}$-linked inhibitor of apoptosis protein (XIAP) and WW domain-containing protein 2 (WWP2) can also ubiquitinate PTEN via K48-dependent polyubiquitin chains. Meanwhile, Shank-interacting protein-like 1 (SIPL1) ubiquitinates PTEN via $\mathrm{K} 63$, which in turn promotes the interaction between SIPL1 and PTEN..$^{45}$ Other modifications, such as Poly-ADP ribosylation, can also affect PTEN localization and stability. ${ }^{46}$ 
A number of proteins are reported to regulate PTEN activity by modifying its conformation, stability, subcellular localization and lipid membrane binding, such as MAGI2, DJ1, MAST2, RhoA-dependent kinase 1 (ROCK1), platelet-derived growth factor receptor (PDGFR). ${ }^{6,11,19,47}$ In addition, Yes-associated protein (YAP), the main downstream target of the mammalian Hippo pathway, could downregulate PTEN by inducing miR-29 to inhibit PTEN translation. As PTEN is an upstream negative regulator of mammalian target of rapamycin (mTOR), YAP can activate mTOR and can promote mTOR-mediated cell proliferation through diminishing PTEN expression. ${ }^{48}$ Notch, an important signal protein in T-cell development, can regulate PTEN expression through Hes1, which may directly repress PTEN promoter activity. ${ }^{49}$

It is well known that miRNAs function in gene expression. The miRNAs that regulate PTEN expression have been extensively studied. So far, more than 40 miRNAs have been proven to regulate the expression of PTEN (Table 1). During viral infection, virus-coding miRNAs can also target PTEN to regulate anti-viral immune responses. ${ }^{50}$

\section{REGULATORY FUNCTIONS OF PTEN IN ADAPTIVE IMMUNITY}

The adaptive immune response is an important process by which immune cells can specifically recognize, process and present antigens to reactive $\mathrm{T}$ cells, which induce $\mathrm{B}$ cells to produce specific antibodies. The adaptive immune response normally includes 3 steps: antigen recognition and processing, the activation of $\mathrm{T}$ and $\mathrm{B}$ cells, and an effective stage. Many types of immune cells are involved in adaptive immunity, including antigen-presenting cells, such as macrophages and dendritic cells (DCs), $\mathrm{T}$ cells and B cells. Recent studies have provided evidence that PTEN plays an important role in the development and the functional regulation of adaptive immune cells. Most of these functions of PTEN are related to its regulation of the $\mathrm{PI} 3 \mathrm{~K}$ signaling pathway.

\section{PTEN AND T CELLS}

During T-cell development, there are many important events occurring, including $\beta$-locus rearrangement, proliferation, T-cell lineage commitment, $\beta$-selection, $\alpha$-locus rearrangement, and negative and positive selection. The PTEN-related PI3K/ AKT pathway is related to many of these events (Figure 2).

Adult hematopoietic stem cells (HSCs) are usually quiescent, but their proliferation rates increase upon exposure to cytokines. During the first stage of HSC development, PTEN is required to maintain quiescent HSCs and to modulate the HSC response to inflammatory cytokines. ${ }^{51}$ Porter et al. ${ }^{52}$ found that Pten-deficient HSCs do not require Granulocyte-colony stimulating factor (G-CSF) to mobilize, although they were hypersensitized to even low doses of exogenous G-CSF.

Juntilla and Koretzky ${ }^{53}$ reported that the PI3K/AKT signaling pathway was important for thymocyte survival and proliferation at the $\beta$-selection checkpoint. Studies in mice with a conditional deletion of PTEN in thymocytes indicate that PI3K may affect V-DJ feedback inhibition during $\beta$-selection. T-cell
Table 1 PTEN-targeting miRNAs in humans

\begin{tabular}{|c|c|c|}
\hline miRNAs & Target sites of the PTEN gene & \# of references \\
\hline miR-10b & Not proven & 1 \\
\hline $\operatorname{miR}-17-5 p$ & 3'UTR: 251-279 & 13 \\
\hline miR-19a-3p & 3'UTR: 1208-1228 & 9 \\
\hline miR-19b-3p & 3'UTR: 1208-1228 & 6 \\
\hline miR-20a-5p & 3'UTR: 251-279 & 3 \\
\hline miR-20b-5p & 3'UTR: 251-279 & 3 \\
\hline miR-21-5p & 3'UTR: 420-437,1587-1608 & 52 \\
\hline miR-22-3p & 3'UTR: 675-696 & 2 \\
\hline miR-23a-3p & 3'UTR: 2266-2286 & 3 \\
\hline miR-23b-3p & 3'UTR: 2266-2286 & 1 \\
\hline miR-25-3p & 3'UTR: 2842-2866 & 1 \\
\hline miR-26a-5p & 3'UTR: 1244-1268, 2605-2626 & 6 \\
\hline miR-26b-5p & 3'UTR: 1244-1268, 2605-2626 & 2 \\
\hline miR-29a & 3'UTR: 681-688, 1741-1747 & 5 \\
\hline miR-32-5p & 3'UTR: 841-2866 & 4 \\
\hline miR-93-5p & 3'UTR: 257-279 & 3 \\
\hline miR-103a-3p & 3'UTR: 1070-1089 & 2 \\
\hline miR-106a-5p & Not proven & 2 \\
\hline miR-106b-5p & Not proven & 4 \\
\hline $\operatorname{miR}-128-3 p$ & Not proven & 2 \\
\hline miR-130a & Not proven & 1 \\
\hline miR-141-3p & 3'UTR: 1453-1474 & 2 \\
\hline miR-144-3p & 3'UTR: 2906-2924 & 1 \\
\hline miR-155-5p & 3'UTR: 329-355 & 3 \\
\hline miR-205-5p & 3'UTR: 487-512 & 3 \\
\hline miR-214-3p & 3'UTR: 1000-1021 & 7 \\
\hline miR-216a-5p & 3'UTR: 1087-1112 & 3 \\
\hline miR-217-3p & 3'UTR: 1313-1335 & 3 \\
\hline miR-221-3p & Not proven & 4 \\
\hline miR-222-3p & Not proven & 5 \\
\hline miR-301a-3p & 3'UTR: 2236-2260 & 1 \\
\hline miR-337 & Not proven & 1 \\
\hline miR-382-5p & 3'UTR: 961-983 & 1 \\
\hline $\operatorname{miR}-425-5 p$ & 3'UTR: 190-214 & 1 \\
\hline miR-429 & 3'UTR: 2500-2523 & 1 \\
\hline miR-494-3p & 3'UTR: 2297-2320 & 2 \\
\hline miR-519a-3p & 3'UTR: 1129-1155 & 2 \\
\hline miR-519c-3p & 3'UTR: 1129-1155 & 1 \\
\hline miR-519d-3p & 3'UTR: 259-279 & 2 \\
\hline miR-543 & Not proven & 1 \\
\hline miR-682 & 3'UTR: 1548-1554 & 1 \\
\hline miR-718 & Not proven & 2 \\
\hline
\end{tabular}

receptor (TCR) $\beta^{-}$cells aberrantly survive in mice that lack both PTEN and the CD3 $\gamma$ chain. Close to half of all doublepositive (DP) cells from $\mathrm{Pten}^{-/-} \mathrm{Cd} 3 \gamma^{-/-}$thymocytes lack intracellular TCR $\beta$, indicating a failure of the $\beta$-selection checkpoint to eliminate cells without productive TCR $\beta^{-}$chain rearrangements. These results suggest that the expansion of TCR $\beta^{-}$cells is likely due to the rescue of cells that should have otherwise been destroyed. Hence, this would contribute to autoimmune disorders and lymphoid hyperplasia. ${ }^{54}$ 


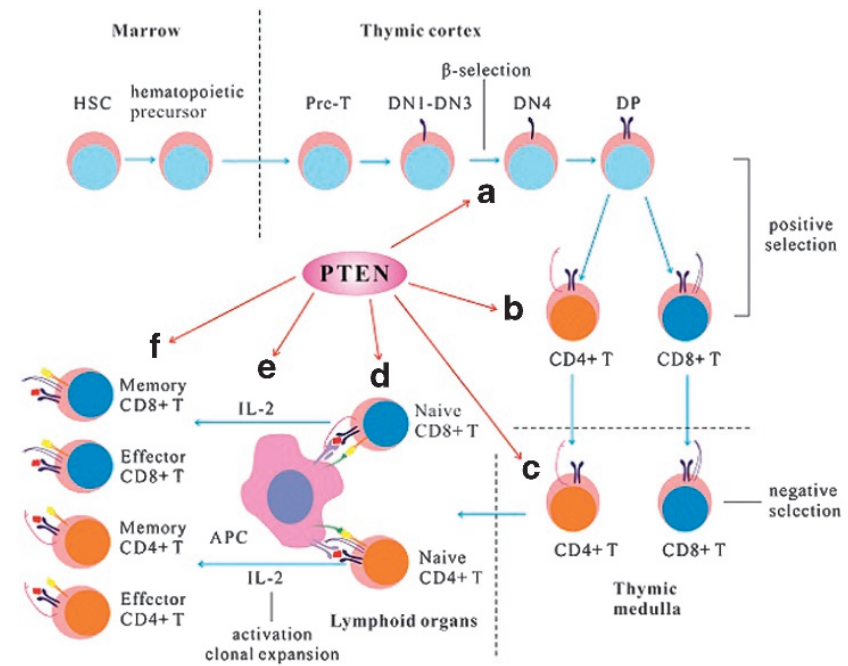

Figure 2 PTEN functions in T-cell development, activation and differentiation. (a) Deletion of PTEN in thymocytes affects V-DJ feedback inhibition during $\beta$-selection; (b) PTEN affects T-cell differentiation. PTEN knock-out in early stages will lead to peripheral lymphomas and autoimmunity; (c) PTEN knockout damages the helper function of CD4+T cells; (d) In PTEN-deleted $\mathrm{T}$ cells, TCR stimulation alone, without CD28 co-stimulation, is sufficient to induce the hyperactivation of the PI3K pathway and to enhance IL-2 production; (e) Upregulation of PTEN expression provides an inhibition signal to reduce IL-2R signaling pathway activation after IL-2 binding; (f) Deletion of PTEN inhibits the development and the survival of memory CD8+ T cells.

During the stem cell stage or the progenitor cell stage, PTEN knockout mice develop peripheral lymphomas and autoimmunity. In CD4+ T cells, PTEN deficiency enhances their helper function but does not lead to autoimmunity or lymphoma. PTEN-deficient Th cells proliferate more rapidly and produce greater levels of cytokines. ${ }^{55}$ However, in terminally differentiated CD8 $+\mathrm{T}$ cells, the deletion of PTEN inhibits the development and the survival of memory CD8 $+\mathrm{T}$ cells by repressing interleukin-7 (IL-7) and IL-15 receptor expression. ${ }^{56}$

PTEN is also important in T-cell activation. Full T-cell activation needs two signals, an antigen-specific TCR engagement signal and a co-stimulatory signal. TCR activation alone initiates T-cell anergy. By contrast, in PTEN knockout CD4+ $\mathrm{T}$ cells, TCR stimulation alone, without CD28 co-stimulation, is sufficient to induce hyperactivation of the PI3K pathway and to enhance IL-2 production. ${ }^{57}$ However, in another study, knockout of all regulatory subunits of class $1 \mathrm{~A}$ PI3K in T cells did not affect T-cell proliferation under the stimulation of two co-stimulatory signals. Its antiviral response remains normal, and its helper function to B-cell activation is impaired. ${ }^{58}$ The disparity of these two findings concerning the role of PTEN in $\mathrm{T}$ cells should be investigated in future studies.

PTEN can negatively regulate the expression of immunosuppressive cytokines, including IL-10, IL-6 and VEGF, in a signal transducer and activator of transcription 3 (STAT3)dependent manner in melanoma cells. Conditioned media from PTEN-deficient melanoma cells can block the production of IL-12 in DC cells. This inhibition can be rescued by restoring PTEN or by using neutralizing antibodies against the immunosuppressive cytokines. Blüml et al..$^{59}$ found that myeloid-specific deletion of PTEN in mice could reduce the expression of IL-23 and IL-6, which are important for Th17 polarization, and consequently affects the development of autoimmune arthritis. PTEN can also downregulate the expression of programmed death-ligand 1 (PD-L1) in melanoma cells. ${ }^{60} \mathrm{PD}-\mathrm{L} 1 \mathrm{can}$ bind with programmed death 1 (PD-1) to transmit an inhibitory signal, which reduces the proliferation of antigen-specific CD8+ $\mathrm{T}$ cells in the lymph node. PTENdeficient carcinoma cells may evade immune responses this way, and the loss of PTEN promotes resistance to T-cellmediated immunotherapy, including the use of anti-PD-1. ${ }^{61,62}$ Moreover, cytokines may affect PTEN functions. For instance, transforming growth factor- $\beta$ (TGF- $\beta$ ) was shown to induce the loss of PTEN enzymatic activity by phosphorylating the $\mathrm{C}$ terminus of PTEN, and tumor necrosis factor- $\alpha$ (TNF- $\alpha$ ) has been shown to increase the expression of PTEN. ${ }^{63}$ Exogenous administration of unphosphorylated PTEN can restore the TGF- $\beta$-induced loss of PTEN activity, and this strategy can be used in the treatment of some TGF- $\beta$-related diseases. ${ }^{47}$

PTEN can influence cytokine-induced responses of the IL-2 receptor by regulating the AKT signaling pathway, which is normally related to the common $\gamma$-chain. $\gamma$-chain is a common subunit of IL-2 receptor subfamily members, which includes receptors for IL-2, IL-4, IL-7, IL-9, IL-15 and IL-21. After activation, the common $\gamma$-chain can transduce signals through three signaling pathways to promote cellular survival and proliferation: the PI3K-AKT pathway, the RAS-MAPK pathway and the JAK-STAT pathway. Upregulation of PTEN expression can provide an inhibitory signal to reduce the activation of the IL-2R signaling pathway after IL-2 binding. ${ }^{64,65}$

PTEN also participates in the immune tolerance of $\mathrm{T}$ cells. Suzuki et al. ${ }^{66}$ found that, in conditional knock-out $P$ ten $^{\text {flox/- }}$ mice, Pten ${ }^{\text {flox/- }}$ T cells hyper-proliferate, are autoreactive, secrete increased levels of Th1/Th2 cytokines, and resist apoptosis. CD4+ $\mathrm{T}$ lymphomas are detectable, and negative selection of peripheral tolerance is impaired in Pten-deficient mice. By contrast, Soond et al. ${ }^{55}$ showed that Pten loss in CD4+ $\mathrm{T}$ cells could not lead to lymphoma. It could be concluded that PTEN deletion in T cells in different maturity stages may lead to different results. This phenomenon could partially explain the mechanisms of some autoimmune diseases and tumorigenesis. ${ }^{66}$

Treg cells are indispensable for regulating the degree of immune response and for preventing the activation of autoreactive $\mathrm{T}$ cells. Bensinger et al. ${ }^{65}$ found that Treg-cell hypoproliferation after IL-2 stimulation is caused by the high expression of PTEN, which can inhibit AKT signaling pathway. Deletion of PTEN can enhance the peripheral homeostasis of Treg cells in vivo and can allow their expansion ex vivo in response to IL-2 alone. ${ }^{64}$ PTEN deficiency in Treg cells can upregulate the activity of mTORC2 and AKT, resulting in exuberant interferon- $\gamma$ (IFN- $\gamma$ ) production, 
excessive $\mathrm{T}_{\mathrm{FH}}$ cell and germinal center response, and spontaneous inflammatory disease. ${ }^{67,68}$ Qiang et al. proved that PTEN can modulate Treg cells and inflammatory responses through the $\beta$-catenin signaling pathway in mouse liver ischemia and reperfusion injury. ${ }^{69}$ Moreover, PTEN can regulate the reciprocal balance between Th17 and Tregs through downregulating STAT3 activation. ${ }^{70}$

\section{PTEN AND B CELLS}

B-cell activation is partially dependent on the intercellular signal transduction of CD19, which is closely related to AKT signal activation. The negative regulation of PTEN to AKT signaling pathways exerts effects on the proliferation, activation and survival of B cells. PTEN governed B-cell subsets, especially B1, follicular B cells, and marginal zone B cells, and was required for immunoglobulin (Ig) classswitching. PTEN-deficient mice have increased numbers of autoantibody-producing B1 B cells. ${ }^{71}$ PTEN also participates in suppressing negative selection and promotes the oncogenic transformation of pre-B cells. ${ }^{72}$

\section{FUNCTIONS OF PTEN IN INNATE IMMUNITY AND ANTIVIRAL DEFENSE}

PTEN is also intricately involved in innate immunity. In humans, innate immune components include innate immune cells, such as macrophages, neutrophils, natural killer (NK) cells, and innate immune molecules, such as pattern recognition receptors (PRRs), proinflammatory cytokines, and IFNs. In addition, nonimmune cells are also involved in innate immunity.

Functions of the PTEN-related AKT signaling pathway in immune and inflammatory responses are controversial. Deletion of PTEN in myeloid cells could enhance macrophage phagocytosis, reduce pulmonary neutrophil influx, diminish TNF- $\alpha$, increase IL-10 and improve survival after S. pneumonia infection. ${ }^{73}$ In addition, PTEN acts as an important regulator of NK cell localization. Leong et al. found that in an NK cellspecific Pten-deletion mouse model, there was a significant expansion of peripheral blood NK cells and the premature egress of NK cells from the bone marrow due to selective hyper-responsiveness to the blood localizing chemokine sphingosine 1-phosphate. PTEN-deleted NK cells were defective at migrating to distal tumor sites but can clear migrated tumor cells in the blood. ${ }^{74}$

For PRR, LPS-challenged PTEN-deficient macrophages display profoundly reduced TNF- $\alpha$ release and diminished activation of MAPKs. PTEN is also responsible for the elevated production of cytokines, such as IL-6, in response to Toll-like receptor (TLR) agonists. Moreover, the deletion of PTEN results in diminished inflammatory responses. PTEN deletion can induce arginase I expression and secretion by increasing the expression of C/EBP $\beta$ and STAT3.$^{75}$ In addition, it can enhance GKS3 $\beta$ activation and thus promote an inflammatory response to TLR agonists in macrophages. ${ }^{73}$ Kamo et al. ${ }^{76}$ found that the knockdown of PTEN could promote AKT/ $\beta$ catenin/Foxol signaling and could down-regulate TLR4 expression in mouse liver cells responding to ischemia/reperfusion injury. The same signaling pathway may also trigger highmobility group box 1-mediated innate immune response in acute lung injury. ${ }^{77}$

Recently, our group demonstrated that PTEN can directly regulate IFN response signaling pathways and thus plays a pivotal role in antiviral innate immunity. ${ }^{53}$ We found that phosphorylation at Ser97 of interferon regulatory factor 3 (IRF3) prevents IRF3 from nuclear import and that PTEN phosphatase can dephosphorylate at Ser97, thus releasing IRF3 from negative regulation (Figure 3). Consequently, knockout of PTEN disrupts nuclear import and the activation of IRF3. We showed that PTEN-depleted cells or PTEN-deficient cancer cells have a low level of type I IFN responses and are more sensitive to virus infection. This type of IFN regulation signaling pathway may affect not only the anti-viral innate immune response but also the anti-tumor effect. ${ }^{78,79}$ This finding may explain why PTEN-deficient tumor cells are more permissive to IFN-sensitive oncolytic viruses and may help to optimize the regimen of viral oncolytic therapies in the future.

IFN also negatively regulates PTEN through interferonstimulated genes (ISGs). For example, ISG-15, a ubiquitinlike modifier, could directly conjugate with PTEN and regulate the stability of PTEN protein. In addition, ubiquitin-specific peptidase 18 (USP18) could help to stabilize PTEN by acting on ISG-15. ${ }^{80}$

\section{PERSPECTIVES}

Since the discovery of PTEN as a tumor suppressor $\sim 20$ years ago, more than 12000 research papers on the functions of PTEN have been published. Although the functions and mechanisms of PTEN in tumor suppression are extensively studied, the roles of PTEN in adaptive and innate immunity have only just begun to be revealed. We believe that studies on

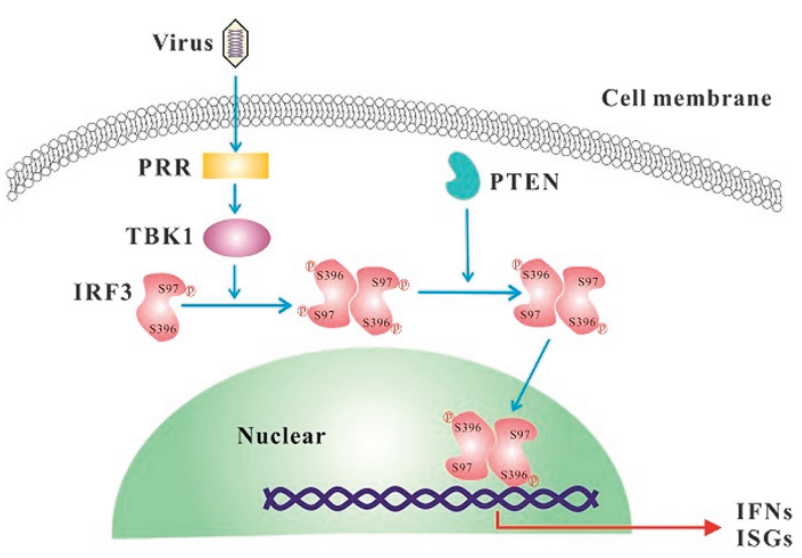

Figure 3 Schematic diagram of the function of PTEN in the dephosphorylation of IRF3 and the regulation of its nuclear import. The S396 site represents a positive phosphorylation site that is essential for IRF3 activation, and the S97 site is a newly identified negative phosphorylation site that controls the nuclear import of IRF3. IFN, interferon; IRF3, interferon regulatory factor 3; ISGs, interferon-stimulated genes; PRR, pattern recognition receptor. 
the function and regulation of PTEN in immune responses will blossom in the future.

Although the principal functions of PTEN are well documented, a number of critical questions about the underlying mechanisms remain to be answered. For example, most domains of PTEN are structured, but it is not fully clear how PTEN forms a dimer and whether there are other proteins involved in the transition between dimer and monomer. Recently, several new substrates of PTEN, such as IRF3 and AKT, have been identified ${ }^{81}$ and indicate that PTEN is involved in the regulation of a complex signaling network. Therefore, other unknown substrates need to be further identified and studied.

As to the immune regulation of PTEN, its roles in T-cell development are well established, and those in B-cell development and innate immune responses are far from unambiguous. The findings by our group highlight that PTEN is critical for IRF3 activation and that the IFN response may represent the tip of the iceberg for functions in innate immunity. Current knowledge of the functions of PTEN in adaptive immunity is mostly limited to the PI3K/AKT signaling pathway. Are there any other pathways involved in adaptive immune responses that are regulated by PTEN? What is the relationship between and crosstalk of those different pathways? Can innate immunity and adaptive immunity affect each other through crosslinking between these pathways? What is the impact of the factors that regulate PTEN function on immune responses? Are there immune molecules that can regulate PTEN and that can function both in tumor suppression and in antiviral immunity? Answers to these questions will deepen our understanding of the functions and regulatory mechanisms of PTEN in antitumor and antiviral responses, which will further benefit the development of PTEN-related therapies of human cancer and viral diseases.

\section{CONFLICT OF INTEREST}

The authors declare no conflict of interest.

\section{ACKNOWLEDGEMENTS}

We acknowledge the financial support provided by the National Natural Science Foundation of China (81620108020 and 31300609) and by Hubei Province's Outstanding Medical Academic Leader Program and Innovation Team (2015CFA009 to DG).

1 Li J, Yen C, Liaw D, Podsypanina K, Bose S, Wang SI et al. PTEN, a putative protein tyrosine phosphatase gene mutated in human brain, breast, and prostate cancer. Science 1997; 275: 1943-1947.

2 Steck PA, Pershouse MA, Jasser SA, Yung WK, Lin H, Ligon AH et al. Identification of a candidate tumour suppressor gene, MMAC1, at chromosome 10q23.3 that is mutated in multiple advanced cancers. Nat Genet 1997; 15: 356-362.

3 Song MS, Salmena L, Pandolfi PP. The functions and regulation of the PTEN tumour suppressor. Nat Rev Mol Cell Biol 2012; 13: 283-296.

4 Worby CA, Dixon JE. PTEN. Annu Rev Biochem 2014; 83: 641-669.

5 Mester J, Charis E. PTEN hamartoma tumor syndrome. Handb Clin Neurol 2015; 132: 129-137.
6 Hopkins BD, Hodakoski C, Barrows D, Mense SM, Parsons RE. PTEN function: the long and the short of it. Trends Biochem Sci 2014; 39: 183-190.

7 Pulido R. PTEN: a yin-yang master regulator protein in health and disease. Methods 2015; 77-78: 3-10.

8 Hopkins BD, Fine B, Steinbach N, Dendy M, Rapp Z, Shaw J et al. A secreted PTEN phosphatase that enters cells to alter signaling and survival. Science 2013; 341: 399-402.

9 Lee JO, Yang H, Georgescu MM, Di Cristofano A, Maehama T, Shi Y et al. Crystal structure of the PTEN tumor suppressor: implications for its phosphoinositide phosphatase activity and membrane association. Cell 1999; 99: 323-334.

10 Heinrich F, Chakravarthy S, Nanda H, Papa A, Pandolfi PP, Ross AH et al. The PTEN tumor suppressor forms homodimers in solution. Structure 2015; 23: 1952-1957.

11 Belotti E, Polanowska J, Daulat AM, Audebert S, Thome V, Lissitzky JC et al. The human PDZome: a gateway to PSD95-Disc large-zonula occludens (PDZ)-mediated functions. Mol Cell Proteomics 2013; 12 2587-2603.

12 Sotelo NS, Schepens JT, Valiente M, Hendriks WJ, Pulido R. PTEN-PDZ domain interactions: binding of PTEN to PDZ domains of PTPN13. Methods 2015; 77-78: 147-156.

13 Takahashi Y, Morales FC, Kreimann EL, Georgescu MM. PTEN tumor suppressor associates with NHERF proteins to attenuate PDGF receptor signaling. EMBO J 2006; 25: 910-920.

14 Jurado S, Benoist M, Lario A, Knafo S, Petrok CN, Esteban JA. PTEN is recruited to the postsynaptic terminal for NMDA receptor-dependent long-term depression. EMBO J 2010; 29: 2827-2840.

15 Rahdar M, Inoue T, Meyer T, Zhang J, Vazquez F, Devreotes PN. A phosphorylation-dependent intramolecular interaction regulates the membrane association and activity of the tumor suppressor PTEN. Proc Natl Acad Sci USA 2009; 106: 480-485.

16 Bolduc D, Rahdar M, Tu-Sekine B, Sivakumaren SC, Raben D, Amzel LM et al. Phosphorylation-mediated PTEN conformational closure and deactivation revealed with protein semisynthesis. eLife 2013; 2: e00691.

17 Nguyen HN, Afkari Y, Senoo H, Sesaki H, Devreotes PN, lijima M. Mechanism of human PTEN localization revealed by heterologous expression in Dictyostelium. Oncogene 2014; 33: 5688-5696.

18 Nakahata S, Ichikawa T, Maneesaay P, Saito Y, Nagai K, Tamura T et al. Loss of NDRG2 expression activates PI3K-AKT signalling via PTEN phosphorylation in ATLL and other cancers. Nat Commun 2014: 5: 3393.

19 Milella M, Falcone I, Conciatori F, Cesta Incani U, Del Curatolo A, Inzerilli $\mathrm{N}$ et al. PTEN: multiple functions in human malignant tumors. Front Oncol 2015; 5: 24.

20 Bassi C, Ho J, Srikumar T, Dowling RJ, Gorrini C, Miller SJ et al. Nuclear PTEN controls DNA repair and sensitivity to genotoxic stress. Science 2013; 341: 395-399.

$21 \mathrm{Gu}$ T, Zhang Z, Wang J, Guo J, Shen WH, Yin Y. CREB is a novel nuclear target of PTEN phosphatase. Cancer Res 2011; 71: 2821-2825.

22 Shen WH, Balajee AS, Wang J, Wu H, Eng C, Pandolfi PP et al. Essential role for nuclear PTEN in maintaining chromosomal integrity. Cell 2007; 128: 157-170.

23 He J, Kang X, Yin Y, Chao KS, Shen WH. PTEN regulates DNA replication progression and stalled fork recovery. Nat Commun 2015; 6: 7620.

24 Johnston SB, Raines RT. Catalysis by the tumor-suppressor enzymes PTEN and PTEN-L. PLoS One 2015; 10: e0116898.

25 Putz U, Howitt J, Doan A, Goh CP, Low LH, Silke J et al. The tumor suppressor PTEN is exported in exosomes and has phosphatase activity in recipient cells. Sci Signal 2012; 5: ra70.

26 Masunaga A, Omatsu M, Kunimura T, Uematsu S, Kamio Y, Kitami A et al. Expression of PTEN and its pseudogene PTENP1, and promoter methylation of PTEN in non-tumourous thymus and thymic tumours. J Clin Pathol 2017; e-pub ahead of print 24 January 2017; doi:10.1136/jclinpath-2016-204220.

27 Roh MR, Gupta S, Park KH, Chung KY, Lauss M, Flaherty KT et al. Promoter methylation of PTEN is a significant prognostic factor in melanoma survival. J Invest Dermatol 2016; 136: 1002-1011.

28 Choi MS, Nakamura T, Cho SJ, Han X, Holland EA, Qu J et al. Transnitrosylation from DJ-1 to PTEN attenuates neuronal cell death in parkinson's disease models. J Neurosci 2014; 34: 15123-15131. 
29 Lee CU, Hahne G, Hanske J, Bange T, Bier D, Rademacher C et al. Redox modulation of PTEN phosphatase activity by hydrogen peroxide and bisperoxidovanadium complexes. Angew Chem Int Ed 2015; 54: 13796-13800.

30 Papa A, Wan L, Bonora M, Salmena L, Song MS, Hobbs RM et al. Cancer-associated PTEN mutants act in a dominant-negative manner to suppress PTEN protein function. Cell 2014; 157: 595-610.

31 Leslie NR, Foti M. Non-genomic loss of PTEN function in cancer: not in my genes. Trends Pharmacol Sci 2011; 32: 131-140.

32 Li Z, Dong X, Wang Z, Liu W, Deng N, Ding Y et al. Regulation of PTEN by Rho small GTPases. Nat Cell Biol 2005; 7: 399-404.

33 Yim EK, Peng G, Dai H, Hu R, Li K, Lu Y et al. Rak functions as a tumor suppressor by regulating PTEN protein stability and function. Cancer Cell 2009; 15: 304-314.

34 Koul D, Jasser SA, Lu Y, Davies MA, Shen R, Shi Y et al. Motif analysis of the tumor suppressor gene MMAC/PTEN identifies tyrosines critical for tumor suppression and lipid phosphatase activity. Oncogene 2002; 21: 2357-2364.

35 Fragoso R, Barata JT. Kinases, tails and more: regulation of PTEN function by phosphorylation. Methods 2015; 77-78: 75-81.

36 Huang J, Yan J, Zhang J, Zhu S, Wang Y, Shi T et al. SUMO1 modification of PTEN regulates tumorigenesis by controlling its association with the plasma membrane. Nat Commun 2012; 3: 911.

$37 \mathrm{Ho}$ J, Bassi C, Stambolic V. Characterization of nuclear PTEN and its post translational modifications. Methods 2015; 77-78: 104-111.

38 Okumura K, Mendoza M, Bachoo RM, DePinho RA, Cavenee WK, Furnari FB. PCAF modulates PTEN activity. J Biol Chem 2006; 281: 26562-26568.

39 Meng Z, Jia LF, Gan YH. PTEN activation through K163 acetylation by inhibiting HDAC6 contributes to tumour inhibition. Oncogene 2016; 35: 2333-2344.

40 Ikenoue T, Inoki K, Zhao B, Guan KL. PTEN acetylation modulates its interaction with PDZ domain. Cancer Res 2008; 68: 6908-6912.

41 Gupta A, Leslie NR. Controlling PTEN (phosphatase and tensin homolog) stability: A DOMINANT ROLE FOR LYSINE 66. J Biol Chem 2016; 291: 18465-18473.

42 Yuan L, LV Y, Li H, Gao H, Song S, Zhang Y et al. Deubiquitylase OTUD3 regulates PTEN stability and suppresses tumorigenesis. Nat Cell Biol 2015; 17: 1169-1181.

43 Lee MS, Jeong MH, Lee HW, Han HJ, Ko A, Hewitt SM et al. PI3K/AKT activation induces PTEN ubiquitination and destabilization accelerating tumourigenesis. Nat Commun 2015; 6: 7769.

44 Leslie NR, Kriplani N, Hermida MA, Alvarez-Garcia V, Wise HM. The PTEN protein: cellular localization and post-translational regulation. Biochem Soc Trans 2016; 44: 273-278.

45 De Melo J, Lin X, He L, Wei F, Major P, Tang D. SIPL1-facilitated PTEN ubiquitination contributes to its association with PTEN. Cell Signal 2014; 26: 2749-2756.

46 Li N, Zhang Y, Han X, Liang K, Wang J, Feng L et al. Poly-ADP ribosylation of PTEN by tankyrases promotes PTEN degradation and tumor growth. Genes Dev 2015; 29: 157-170.

47 Kimura M, Hashimoto N, Kusunose M, Aoyama D, Sakamoto K, Miyazaki $\mathrm{S}$ et al. Exogenous induction of unphosphorylated PTEN reduces TGFbeta-induced extracellular matrix expressions in lung fibroblasts. Wound Repair Regen 2016; 25: 86-97.

48 Tumaneng K, Schlegelmilch K, Russell RC, Yimlamai D, Basnet H, Mahadevan N et al. YAP mediates crosstalk between the Hippo and PI (3)K-TOR pathways by suppressing PTEN via miR-29. Nat Cell Biol 2012; 14: 1322-1329.

49 Wong GW, Knowles GC, Mak TW, Ferrando AA, Zuniga-Pflucker JC. HES1 opposes a PTEN-dependent check on survival, differentiation, and proliferation of TCRbeta-selected mouse thymocytes. Blood 2012; 120: 1439-1448.

50 Bernhardt K, Haar J, Tsai MH, Poirey R, Feederle R, Delecluse HJ. A viral microRNA cluster regulates the expression of PTEN, p27 and of a bcl-2 homolog. PLoS Pathog 2016; 12: e1005405.

51 Lee JY, Nakada D, Yilmaz OH, Tothova Z, Joseph NM, Lim MS et al. mTOR activation induces tumor suppressors that inhibit leukemogenesis and deplete hematopoietic stem cells after PTEN deletion. Cell Stem Cell 2010; 7: 593-605.

52 Porter SN, Cluster AS, Signer RA, Voigtmann J, Monlish DA, Schuettpelz LG et al. PTEN cell autonomously modulates the hematopoietic stem cell response to inflammatory cytokines. Stem Cell Rep 2016; 6: 806-814.
53 Juntilla MM, Koretzky GA. Critical roles of the PI3K/Akt signaling pathway in T cell development. Immunol Lett 2008; 116: 104-110.

54 Buckler JL, Liu X, Turka LA. Regulation of T-cell responses by PTEN. Immunol Rev 2008; 224: 239-248.

55 Soond DR, Garcon F, Patton DT, Rolf J, Turner M, Scudamore C et al. Pten loss in CD4 T cells enhances their helper function but does not lead to autoimmunity or lymphoma. J Immunol 2012; 188: 5935-5943.

56 Hand TW, Cui W, Jung YW, Sefik E, Joshi NS, Chandele A et al. Differential effects of STAT5 and PI3K/AKT signaling on effector and memory CD8 T-cell survival. Proc Natl Acad Sci USA 2010; 107: 16601-16606.

57 Buckler JL, Walsh PT, Porrett PM, Choi Y, Turka LA. Cutting edge: T cell requirement for $\mathrm{CD} 28$ costimulation is due to negative regulation of TCR signals by PTEN. J Immunol 2006; 177: 4262-4266.

58 Deane JA, Kharas MG, Oak JS, Stiles LN, Luo J, Moore TI et al. T-cell function is partially maintained in the absence of class IA phosphoinositide 3-kinase signaling. Blood 2007; 109: 2894-2902.

59 Bluml S, Sahin E, Saferding V, Goncalves-Alves E, Hainzl E, Niederreiter B et al. Phosphatase and tensin homolog (PTEN) in antigen-presenting cells controls Th17-mediated autoimmune arthritis. Arthritis Res Ther 2015; 17: 230.

60 Dong Y, Richards JA, Gupta R, Aung PP, Emley A, Kluger Y et al. PTEN functions as a melanoma tumor suppressor by promoting host immune response. Oncogene 2014; 33: 4632-4642.

61 George S, Miao D, Demetri GD, Adeegbe D, Rodig SJ, Shukla S et al. Loss of PTEN is associated with resistance to anti-PD-1 checkpoint blockade therapy in metastatic uterine leiomyosarcoma. Immunity 2017; 46: 197-204.

62 Peng W, Chen JQ, Liu C, Malu S, Creasy C, Tetzlaff MT et al. Loss of PTEN promotes resistance to T cell-mediated immunotherapy. Cancer Discov 2016; 6: 202-216.

63 da Costa RM, Neves KB, Mestriner FL, Louzada-Junior P, Bruder-Nascimento T, Tostes RC. TNF-alpha induces vascular insulin resistance via positive modulation of PTEN and decreased Akt/eNOS/ NO signaling in high fat diet-fed mice. Cardiovasc Diabetol 2016; 15: 119.

64 Walsh PT, Buckler JL, Zhang J, Gelman AE, Dalton NM, Taylor DK et al. PTEN inhibits IL-2 receptor-mediated expansion of CD4+ CD25 + tregs. J Clin Invest 2006; 116: 2521-2531.

65 Bensinger SJ, Walsh PT, Zhang J, Carroll M, Parsons R, Rathmell JC et al. Distinct IL-2 receptor signaling pattern in CD4+CD25+ regulatory T cells. J Immunol 2004; 172: 5287-5296.

66 Suzuki A, Yamaguchi MT, Ohteki T, Sasaki T, Kaisho T, Kimura Y et al. T cell-specific loss of PTEN leads to defects in central and peripheral tolerance. Immunity 2001; 14: 523-534.

67 Ray JP, Craft J. PTENtiating autoimmunity through Treg cell deregulation. Nat Immunol 2015; 16: 139-140.

68 Shrestha S, Yang K, Guy C, Vogel P, Neale G, Chi H. Treg cells require the phosphatase PTEN to restrain $\mathrm{TH} 1$ and TFH cell responses. Nat Immunol 2015; 16: 178-187.

69 Zhu Q, Li C, Wang K, Yue S, Jiang L, Ke M et al. PTEN-beta-catenin signaling modulates regulatory $\mathrm{T}$ cells and inflammatory responses in mouse liver ischemia and reperfusion injury. Liver Transp/ 2017; e-pub ahead of print 2 February 2017; doi:10.1002/lt.24735.

70 Lee SH, Park JS, Byun JK, Jhun J, Jung K, Seo HB et al. PTEN ameliorates autoimmune arthritis through down-regulating STAT3 activation with reciprocal balance of Th17 and Tregs. Sci Rep 2016; 6: 34617.

71 Suzuki A, Kaisho T, Ohishi M, Tsukio-Yamaguchi M, Tsubata T, Koni PA et al. Critical roles of PTEN in B cell homeostasis and immunoglobulin class switch recombination. J Exp Med 2003; 197: 657-667.

72 Shojaee S, Chan LN, Buchner M, Cazzaniga V, Cosgun KN, Geng H et al. PTEN opposes negative selection and enables oncogenic transformation of pre-B cells. Nat Med 2016; 22: 379-387.

73 Schabbauer G, Matt U, Gunzl P, Warszawska J, Furtner T, Hainzl E et al. Myeloid PTEN promotes inflammation but impairs bactericidal activities during murine pneumococcal pneumonia. J Immunol 2010; 185: 468-476.

74 Leong JW, Schneider SE, Sullivan RP, Parikh BA, Anthony BA, Singh $A$ et al. PTEN regulates natural killer cell trafficking in vivo. Proc Natl Acad Sci USA 2015; 112: E700-E709.

75 Sahin E, Haubenwallner S, Kuttke M, Kollmann I, Halfmann A, Dohnal AM et al. Macrophage PTEN regulates expression and secretion of arginase I modulating innate and adaptive immune responses. J Immunol 2014; 193: 1717-1727. 
76 Kamo N, Ke B, Busuttil RW, Kupiec-Weglinski JW. PTEN-mediated Akt/beta-catenin/Foxol signaling regulates innate immune responses in mouse liver ischemia/reperfusion injury. Hepatology 2013; 57: 289-298.

77 Zhou M, Zhang Y, Chen X, Zhu J, Du M, Zhou L et al. PTEN-Foxo1 signaling triggers HMGB1-mediated innate immune responses in acute lung injury. Immunol Res 2015; 62: 95-105.

$78 \mathrm{Li} \mathrm{S}$, Zhu M, Pan R, Fang T, Cao YY, Chen S et al. The tumor suppressor PTEN has a critical role in antiviral innate immunity. Nat Immunol 2016; 17: 241-249.
79 Champion BR, Fisher K, Seymour L. A PTENtial cause for the selectivity of oncolytic viruses? Nat Immunol 2016; 17: 225-226.

80 Mustachio LM, Kawakami M, Lu Y, Rodriguez-Canales J, Mino B, Behrens $C$ et al. The ISG15-specific protease USP18 regulates stability of PTEN. Oncotarget 2017; 8: 3-14.

81 Phadngam S, Castiglioni A, Ferraresi A, Morani F, Follo C, Isidoro C. PTEN dephosphorylates AKT to prevent the expression of GLUT1 on plasmamembrane and to limit glucose consumption in cancer cells. Oncotarget 2016; 7: 84999-85020. 